\title{
ARTICLE
}

Clinical Research

\section{Changes in cerebral metabolic activity in men undergoing androgen deprivation therapy for non-metastatic prostate cancer}

\author{
M. M. Cherrier ${ }^{1}$ D. J. Cross $^{2}$ - C. S. Higano ${ }^{3}$ S. Minoshima ${ }^{2}$ \\ Received: 30 October 2017 / Revised: 13 December 2017 / Accepted: 18 December 2017 / Published online: 27 April 2018 \\ (c) The Author(s) 2018. This article is published with open access
}

\begin{abstract}
Objective Androgen deprivation therapy (ADT) is a common treatment option for men with biochemical relapse from prostate cancer. ADT is associated with changes in mood, cognition, and quality of life, and most recently with increased risk for Alzheimer's disease (AD). This study examined changes in brain metabolism using positron emission tomography (PET) in men undergoing intermittent ADT.

Methods Nine men with prostate cancer and a rising PSA (biochemical recurrence) without evidence of metastases were treated with intermittent ADT consisting of 9 months of complete androgen blockade achieved with combined leuprolide acetate and flutamide. Patients underwent resting [Fuorine-18] fluorodeoxyglucose PET ( ${ }^{18}$ F-FDGPET) at baseline (before treatment) and again after 9 months of ADT.

Results Whole-brain mapping analysis after 9 months of androgen deprivation compared to pretreatment baseline revealed decreased regional cerebral glucose metabolism in the cerebellum, posterior cingulate, and medial thalamus bilaterally. Associations of brain metabolism with measurements of cognition and mood while on androgen deprivation revealed positive correlations between the posterior cingulate, left inferior parietal lobule (BA40), and left mid temporal gyrus (BA39) and spatial reasoning and a negative correlation between left inferior parietal lobule and verbal memory. Several mood indices were negatively correlated with hypothalamus and brainstem.

Conclusion These findings suggest that complete androgen deprivation may result in changes in regional brain metabolism associated with variation in mood, verbal memory, and spatial performance. Brain regions that were impacted from ADT are similar and overlap with brain regions with metabolic decline found in early AD and diabetes, suggesting possible common mechanisms.
\end{abstract}

\section{Introduction}

Prostate cancer is the most common form of non-skin cancer diagnosed in men in the United States. Androgen deprivation therapy (ADT) is a common treatment option for men with localized prostate cancer (e.g., in combination with radiation therapy) or for those with a rising PSA after

M. M. Cherrier

cherrier@u.washington.edu

1 Department of Psychiatry and Behavioral Sciences, University of Washington School of Medicine, Seattle, WA, USA

2 Department of Radiology and Imaging Sciences, University of Utah, School of Medicine, Salt Lake City, UT, USA

3 Department of Medicine, Division of Oncology and Urology, University of Washington School of Medicine, Seattle, WA, USA definitive therapy without evidence of metasases. In contrast to those with metastatic disease, such patients have no or minimal disease-related symptoms and may live for many years.

Physiological consequences of ADT may include obesity, anemia, loss of bone mineral density, muscle atrophy, hot flashes, gynecomastia, diabetes, and metabolic syndrome [1]. Changes in quality of life in men undergoing ADT have been previously reported including changes in mood and subjective symptoms of cognitive difficulties [2].

Recent population-based studies have suggested that men undergoing ADT are twice as likely to develop Alzheimer's disease within a few years of initiating ADT [3-5]. While not all studies support this connection [6], there is an indication that ADT decreases cognitive function [7-9], brain function [10], and neuronal structure in humans, all of which are prodromal indices of progressive cognitive decline and possible AD [11]. The disease mechanism of 
$\mathrm{AD}$ is unknown, and complex. However, several side effects and impacts of ADT are known to be involved in proposed $\mathrm{AD}$ disease mechanisms including loss of testosterone and estradiol [12-15], inflammatory processes, disruptions to the immune system $[12,16]$, abnormal lipid and glucose metabolism $[17,18]$, reduced physical activity [19, 20], and disrupted sleep impacting the glymphatic system (a possible method for brain toxin clearance) [21, 22].

The aim of this study was to determine if men undergoing intermittent ADT evidence changes in brain metabolism as measured by fluorine-18 fluorodeoxyglucose $\left({ }^{18} \mathrm{~F}-\mathrm{FDG}\right)$ positron emission tomography (PET) imaging. ${ }^{18}$ F-FDGPET imaging is a sensitive method for measuring changes in metabolic changes in the brain, and has been shown to be a reliable method for detecting early declines in brain function in Alzheimer's disease that corresponds to declines in cognitive and daily function as well as neuropathology [23]. Participants were a subset of men enrolled in a study examining mood and cognitive changes from ADT [7]. We examined patients prospectively, taking advantage of the intermittent aspect of ADT to include a baseline before treatment and repeated neuroimaging after 9 months of ADT, while still on the treatment. Previous findings of declines in spatial reasoning abilities [24] and studies relating parietal and occipital regions involved in spatial processing [25] led us to hypothesize that complete androgen blockade would alter metabolism in parietal and occipital brain regions.

\section{Materials and methods}

\section{Participants}

Patients were recruited from the genitourinary oncology clinic at the Seattle Cancer Care Alliance and the Urology clinics at the University of Washington Medical Center. Participants were part of a larger study examining cognitive and mood changes from ADT [7], willing to undergo the additional neuroimaging procedures, and met additional criteria for PET imaging. Physicians and nurses informed patients of the opportunity to participate and if they demonstrated interest, they contacted the study coordinator who conducted a brief phone screen to determine eligibility. In order to be eligible for treatment with ADT, patients had to have a rising prostate specific antigen (PSA) following primary therapy (prostatectomy, external beam radiation, or brachytherapy) without evidence of metastatic disease by imaging on bone scan and computed tomography scan of the chest, abdomen, and pelvis. Patients who had pain due to prostate cancer, prior psychiatric illness involving hospitalization, dementia, central nervous system metastasis, history of systemic chemotherapy, and current renal or hepatic dysfunction or diabetes or previous brain injury $(>1$ $\mathrm{h}$ loss of consciousness), surgery or known brain lesion or anomaly that would interfere with PET procedures were not eligible. All participants signed written informed consent approved by the University of Washington Institutional Review Board prior to study procedures.

\section{Study design}

Patients received ADT consisting of an anti-androgen (flutamide $250 \mathrm{mg}$ TID) and a GnRH analog (leuprolide acetate $7.5 \mathrm{mg}$ IM (intramuscular) monthly for a total of 9 months duration whereupon ADT was stopped. Patients underwent resting ${ }^{18} \mathrm{~F}$-FDG-PET at baseline before the start of treatment and again after 9 months of ADT. Blood samples for total testosterone $(\mathrm{T})$ and estradiol $\left(\mathrm{E}_{2}\right)$ were also obtained at the same intervals.

\section{Cognitive and mood measures}

Tests included a complex spatial design task in which participants construct complex designs using component parts and the task is timed to completion [26], a mental rotation task in which participants were asked to match complex figures that had been rotated, and a higher score indicates better performance [27]. Verbal memory tasks included a list learning test in which participants heard a list of words read to them followed by a short delay and recall of the list [28], and a story recall task in which participants heard a short story read to them followed by a short and long delay recall [29]. A verbal fluency test, in which participants were required to name as many words beginning with a particular letter in $60 \mathrm{~s}$ [30]. For the Stroop test, participants were timed and asked to read words, color blocks, or name the ink color of printed words [31]. On the self-ordered pointing test (SOPT), participants were required to remember an increasing number of visual designs presented in a changing array [32]. Mood assessment included the profile of mood states (POMS) that utilizes a set of mood adjectives and asks participants to rate mood over the past week using a Likert scale. Subscales include tension-anxiety (TA), depression-dejection (Dep), anger-hostility ( $\mathrm{AH})$, vigor-activity (VA), fatigue-inertia (FI), and confusion-bewilderment (CON) [33]. Tests and questionnaires are described in additional detail in Cherrier et al. [7].

\section{${ }^{18}$ F-FDG-PET image acquisition and analysis}

After intravenous injection of $370 \mathrm{MBq}$ [Fluorine-18]FDG and 45 min uptake at rest in a quiet room with eyes open, standard ${ }^{18}$ F-FDGPET brain imaging (20-min emission scan and 25-min germanium-68 transmission scan for attenuation correction) in a GE Advance scanner (GE 
Medical Systems, Milwaukee, Wisconsin), was performed for each subject. Images were reconstructed to in-plane resolution of $\sim 6 \mathrm{~mm}$ (full-width-half-maximum). PET image sets were co-registered and anatomically standardized to the human brain atlas [34] using NEUROSTAT (University of Utah) [35, 36]. Pixel intensities were normalized to global cortical activity (set to 1000) and images were smoothed with a Gaussian kernel with $\sigma=2.25 \mathrm{~mm}$ to minimize residual anatomic differences across subjects. To obtain regional values for metabolic activity from a standardized template, PET images were also processed using quantitative data extraction algorithm, three-dimensional stereotactic surface projections (3D-SSPs) [37, 38].

\section{Statistical mapping}

Regional metabolic changes pre- and post ADT were assessed using voxelwise one-sample $t$ statistics and probability integral conversion to $z$-scores. Based on the number of voxels and smoothness of the statistical map, a type I error rate was controlled at 0.05 to account for multiple comparisons [39]. To confirm voxelwise analyses, independent analysis using stereotactically predefined volumes of interest was also performed. In this analysis, stereotactically predefined regions (medial and lateral frontal, parietal, and temporal association cortices and precuneus/posterior cingulate) were applied to the standardized and normalized 3D-SSP data sets as described previously [37, 38]. To evaluate the association of cognition and mood with metabolic activity after ADT, the individual values obtained from the tests were used in a voxelwise correlation analysis of the FDG-PET images over the entire cohort. Correlation coefficients for each voxel were converted to $Z$-scores (Fisher transformation) and peak locations of significant correlations were mapped over the entire brain. Regional metabolic changes at structures were examined using a paired $t$ test and general linear model controlling age as covariates for change over time. For all statistical analyses, peaks with $Z$-scores $\geq 4.0$ were considered significant, controlling the type I error rate approximately at $p=0.05$ after correction for multiple comparisons based on image smoothness and voxel number. This $Z$ and $p$ value were selected using established theory of statistical analysis methods for PET images [40, 41].

\section{Hormone analyses}

A secondary analysis was performed to assess changes in circulating hormone levels testosterone $(\mathrm{T})$ and estradiol $\left(E_{2}\right)$ in response to treatment. Serum samples were taken during clinic visits at baseline, and on treatment and were sent to the UWMC clinical laboratory. A repeatedmeasure analysis of variance (ANOVA) with total testosterone and estradiol was used to assess change from baseline. Information on statistical scripts, data, and imaging analysis can be found at http://www.osf.io/ 3yxdu.

\section{Results}

Nine ADT patients completed the screening visit and were eligible to participate. One patient did not complete a second imaging visit due to a scheduling conflict. Only participants who completed all visits were included in the analyses. ADT patients ranged in age from 50 to 75 years with a mean age of 64 years. Education ranged from 12 to 17 years with a mean education level of 14.6 years. All participants were

Table 1 Demographics, serum hormone values, neuropsychological and mood assessment scores

\begin{tabular}{lll}
\hline & Mean (SD) & Mean (SD) \\
\hline Demographics & & \\
Age (years) & $64.0(8.4)$ & \\
Education (years) & $14.6(2.4)$ & \\
& Baseline & 9 months \\
Testosterone (ng/ml) & $3.37(0.77)$ & $0.22(0.07)$ \\
Estradiol (pg/ml) & $27.0(7.2)$ & $22.1(4.48)$ \\
Spatial memory (route test) & $16.71(7.67)$ & $21.57(10.62)$ \\
Spatial ability (block design) & $9.0(0.0)$ & $8.85(0.37)$ \\
Spatial ability (mental rotation) & $7.28(1.38)$ & $6.71(2.28)$ \\
Verbal memory (proactive & $20.57(4.79)$ & $21.21(4.96)$ \\
interference) & & \\
Verbal memory (story recall) & $32.46(10.26)$ & $39.28(9.17) *$ \\
Verbal ability (verbal fluency) & $30.00(2.38)$ & $27.42(7.18)$ \\
Executive function (Stroop) & $42.28(5.49)$ & $46.42(9.18)$ \\
Executive function (SOPT) & $11.28(6.70)$ & $11.42(8.48)$ \\
POMS: aggression and hostility & $45.85(5.49)$ & $47.57(5.53)$ \\
POMS: vigor & $60.71(7.97)$ & $58.4(10.56)$ \\
POMS: concentration & $48.57(9.84)$ & $49.42(8.46)$ \\
POMS: tension and anxiety & $46.71(8.78)$ & $47.14(8.61)$ \\
POMS: fatigue & $50.00(11.0)$ & $54.28(10.90)$ \\
POMS: depression & $46.14(4.67)$ & $48.14(6.54)$ \\
\hline
\end{tabular}

*Verbal memory story recall—number of correctly recalled bits of information after a delay $p=0.02$; verbal ability verbal fluencynumber of words generated for two letters; executive function Stroop - time to complete the interference trial; executive function selfordered pointing test (SOPT)-number of errors summed across all trials. Profile of mood state (POMS) subscales

**Testosterone $\mathrm{ng} / \mathrm{ml}$ change from baseline $p=0.00$, esradiol $\mathrm{pg} / \mathrm{ml}$, spatial memory route test-number of correctly recalled sequences after a delay; spatial ability block design—number of correctly completed designs; spatial ability mental rotation-number of correctly identified figures; verbal memory proactive interferencenumber of correctly recalled words summed across four trials 
Caucasian, non-hispanic. See Table 1 for demographics, hormone, cognitive, and mood data.

\section{Hormone analyses}

A repeated-measure ANOVA revealed a significant change over time for total testosterone $F(1,6)=115.9$, $p<0.01$ and changed from $3.37 \quad(0.77) \mathrm{nmol} / \mathrm{l}$ at baseline to $0.22(0.07)$ at month 9 . Estradiol decreased from 27.0 (7.2) to $22.1(4.48) \mathrm{pmol} / \mathrm{l}$, but this was not a significant decrease.

\section{Voxelwise analysis of pre-/post-ADT}

Whole-brain analysis of pretreatment (pre) compared to on ADT (post) changes in metabolism revealed significant declines cerebellum (4.2\% decreased, $Z=-5.09, p=$ 0.048 ), posterior cingulate ( $3 \%$ decreased, $Z=-4.58, p=$ $0.021)$, and medial thalamus (bilateral) $(4.8 \%$ decreased, $Z$ $=-3.95, p=0.006, p=0.005$ for right and left, respectively) (Fig. 1).

\section{Metabolic activity associated with cognition while on ADT}

Cortical glucose metabolism was associated positively and negatively with select cognitive tasks. Correlation analysis indicated a positive association between performance on a spatial reasoning task (mental rotation) and the posterior cingulate (PCing), left inferior parietal lobule (LPi) (BA40), and left mid temporal lobe (BA39). Higher scores on the mental rotation task indicate better performance. A positive association was observed between the (Stroop test) (higher score is better performance) and activation in left middle frontal gyrus (GFm) (BA 6) and precuneus $(\mathrm{PCu})(\mathrm{BA} 7)$ (see Fig. 2).

Performance on a verbal memory task (story recall) and a (verbal list learning task) revealed a negative association between left inferior parietal lobule and BA 8 and 9. A higher score on both of these verbal memory tasks indicates better performance. A negative association for inferior parietal lobule, supramarginal gyrus, superior temporal gyrus, fusiform, and bilateral cerebellum was found for the Stroop test (higher score indicates better performance) (see Fig. 3).

Cortical brain activity was associated negatively with mood. Scores on the POMS that measures mood were negatively correlated with the brainstem and hypothalamus including subscales of depression (DEP), tension/anxiety (TA), and aggression/hostility (AH). The subscale scores of the POMS are compared to normative values, which allows for generation of $t$ tests. The direction of the scales are such that a higher score indicates more severe depression symptoms (DEP), and more symptoms of tension and anxiety (TA), and higher levels of aggression and hostility (AH) (see Fig. 4).

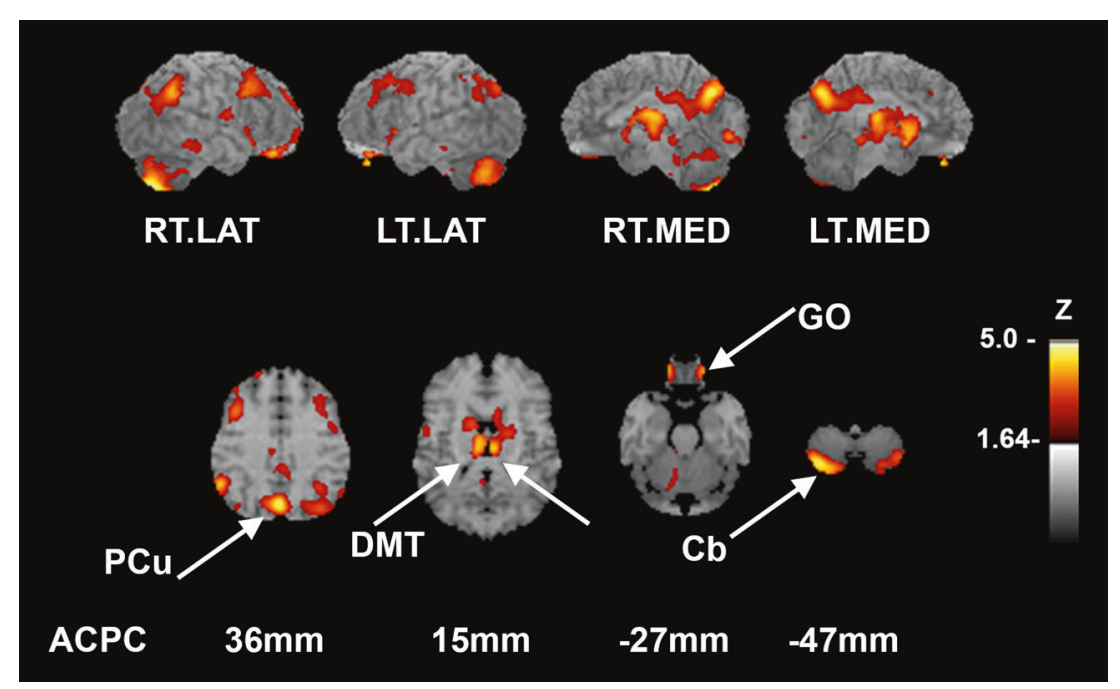

Fig. 1 Voxelwise comparison of pre- and post-ADT treatment reveals resting hypometabolic regions (i.e. regions showing a significant decrease from baseline). Refer to color bar on the right that indicates the corresponding $\mathrm{Z}$-score of change to color, with lighter color indicating a larger change in $\mathrm{Z}$ score. Top row indicates the pattern of hypometabolic deficits on surface projected $z$-score maps. In subjects, pre- and post-FDG-PET images were compared in a voxelwise paired subtraction analysis and $p$ values converted to $Z$-scores. Resultant $Z$ - score map was superimposed to an anatomical MRI template for better visualization of hypometabolic pattern. Sagittal aspects are noted. Second row indicates relevant structures identified on axial slices in $\mathrm{mm}$ from the AC-PC line according to the stereotactic atlas [14]. ( $p<0.05$ after controlling type I error rate for multiple comparisons). RT.LAT right lateral, LT.LAT left lateral, RT.MED right medial, LT. MED left medial, $\mathrm{PCu}$ precuneus, DMT dorsomedial thalamus, $\mathrm{Cb}$ cerebellum 


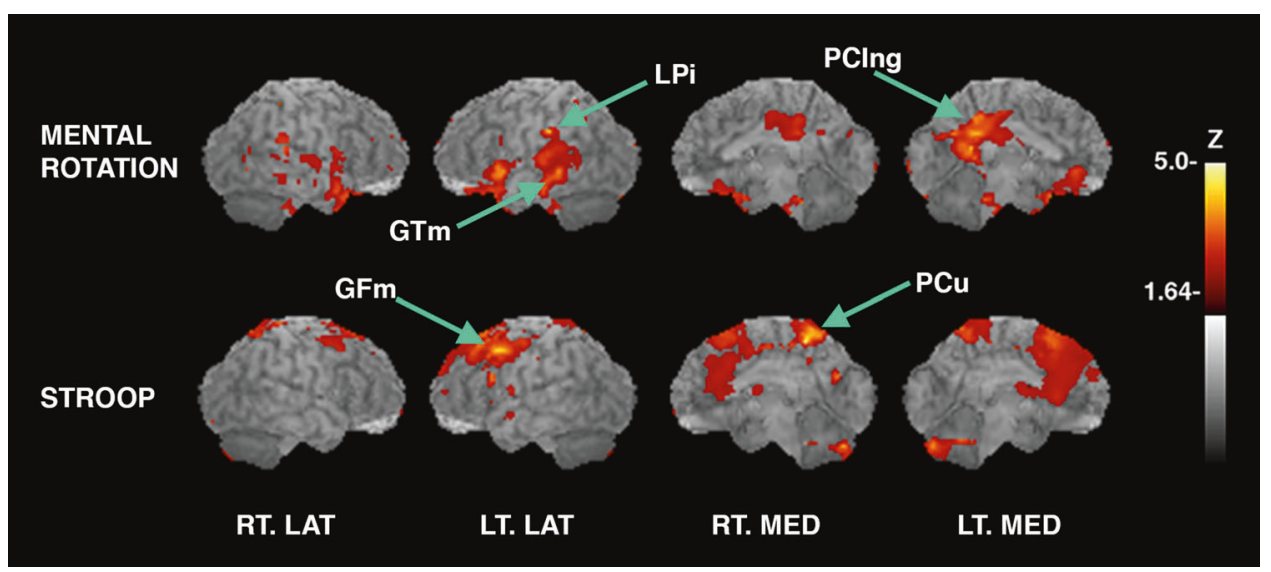

Fig. 2 Surface projected maps of glucose metabolism correlated to tasks with correlation coefficients converted to $Z$-scores and superimposed to structural MRI. Significant structures are as indicated by arrows and labels. Sagittal aspects are noted. Upper panel: Voxelwise linear regression of score on a spatial reasoning task with FDG-PET images across all subjects reveals significant peaks in structures including PCing: posterior cingulate, $z=4.2$, left LPi: inferior parietal lobule, $z=4.1$ and, left GTm: middle temporal gyrus, 4.0. Lower panel: Voxelwise linear regression of score on the Stroop test with FDG-PET images reveals significantly correlated peaks in the PCu: precuneus, $z=4.9$ and the left GFm: middle frontal gyrus, $z=4.1$. ( $p$ $<0.05$ after controlling type I error rate for multiple comparisons). RT LAT right lateral, LT.LAT left lateral, RT.MED right medial, LT. MED left medial

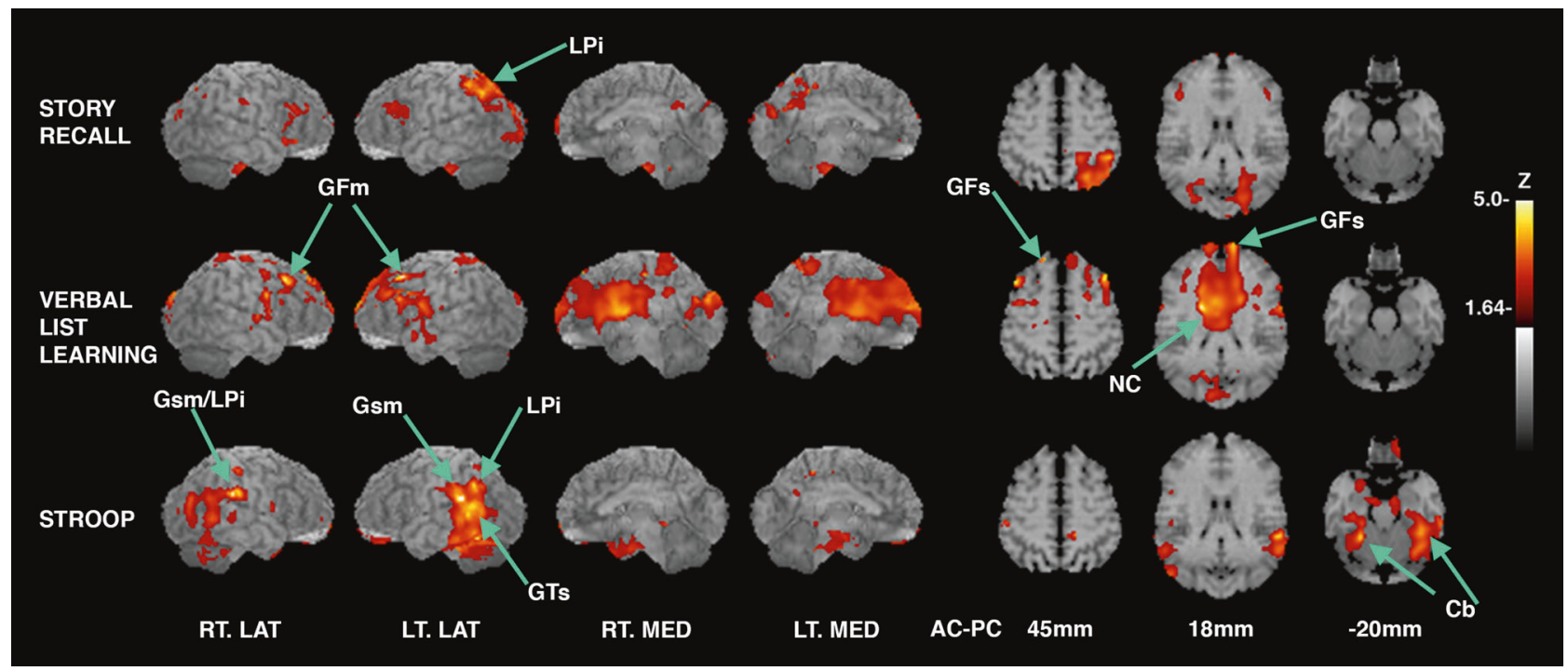

Fig. 3 Cortical glucose metabolism negatively associated with cognition. Surface projected maps of glucose metabolism correlated to tasks with correlation coefficients converted to Z-scores and superimposed to structural MRI. Significant peaks are as indicated by arrows and labels. Sagittal aspects are noted. Relevant structures also identified on axial slices (right side) in $\mathrm{mm}$ from the AC-PC line according to the stereotactic atlas. Upper panel (left): Voxelwise linear regression of score on a story task with FDG-PET images across all subjects reveals a single significant peak in the left LPi: inferior parietal lobule, $z=-4.4$. Middle panel (left): Voxelwise linear regression of score on the verbal list learning task with FDG-PET images reveals significantly correlated peaks in the left GFm: middle frontal gyrus, $z=-5.0$, right NC: caudate, $Z=-5.0$, and bilateral GFs: superior frontal gyrus, $Z=-5.0$. Lower panel (left) Voxelwise linear regression of score on the Stroop test with FDG-PET images reveals significantly correlated peaks in the bilateral LPi: inferior parietal lobule, $z=-5.7$, Gsm: supramarginal gyrus, $z=-4.3$, and $\mathrm{Cb}$ : cerebellum, $z=-4.2$ and in the left GTs: superior temporal gyrus, $z=-5.5$. $(p<0.05$ after controlling type I error rate for multiple comparisons). RT.LAT right lateral, LT.LAT left lateral, RT.MED right medial, LT.MED left medial

\section{Discussion}

Our findings indicate that otherwise healthy men, with nonmetastatic prostate cancer undergoing intermittent ADT demonstrate decreases in brain metabolism in posterior cingulate, cerebellum, and thalamus (dorsal medial) (Fig. 1). We are not aware of a prior report of changes in brain metabolism in response to ADT in humans, in which the state of castration was compared to the eugonadal baseline using a within subject design. Brain regions 


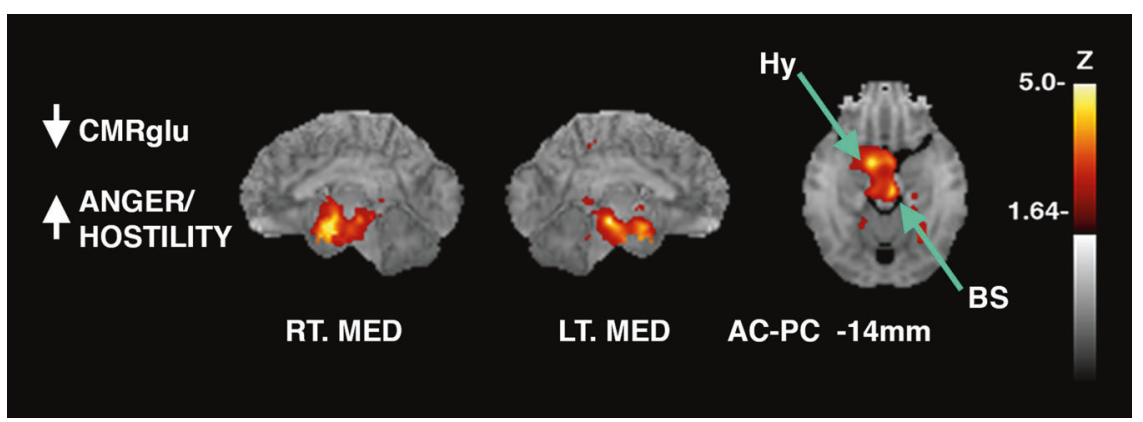

Fig. 4 Surface projected maps of glucose metabolism correlated to tasks with correlation coefficients converted to $Z$-scores and superimposed to structural MRI. Significant peaks are as indicated by arrows and labels. Sagittal aspects are noted. Relevant structures also identified on axial slices in $\mathrm{mm}$ from the AC-PC line according to the stereotactic atlas [14]. Voxelwise linear regression of scores on the
POMS (profile of mood state) that measures mood with FDG-PET images across all subjects reveals significant peaks in the BS: brainstem, $z=-5.0$ and Hy: hypothalamus, $z=-4.6$ ( $z$-score map for anger/hostility shown). ( $p<0.05$ after controlling type I error rate for multiple comparisons). RT.LAT right lateral, LT.LAT left lateral, RT. MED right medial, LT.MED left medial showing decline in metabolism from ADT overlap with brain regions with metabolic decline from Alzheimer's disease $(\mathrm{AD})$ as well as type-2 diabetes [42, 43].

The pathogenesis of $\mathrm{AD}$ is complex and multi-factorial. However, there are possible reasons for concern of an $\mathrm{AD}$ and ADT connection. Testosterone and estradiol, suppressed by ADT have been previously shown in vivo in animal models of $\mathrm{AD}$ and in humans to change serum amyloid-beta (A $\beta$ ) levels and accumulation, which has a critical role in amyloid plaque formation and neuronal death [12-15].

Ding et al. [13] examined the impact of loss of estrogen via ovariectomy $(\mathrm{OVX})$ in a triple-transgenic mouse model of AD. OVX resulted in decreased brain glucose uptake and increased abeta oligomer levels, which was reversed with 17- $\beta$ estradiol administration. Amyloid plaques along with neurofibrillary tangles are the major neuropathological findings in $\mathrm{AD}$ that provide for post-mortem confirmation of disease. Gonadotropin releasing hormone $(\mathrm{GnRH})$ receptors, in the hypothalamus, are part of the homeostatic control for releasing hormones and gonadal steroids. GnRH receptors activate immune cells that have been shown to influence amyloid-beta and amyloid brain plaques $[12,16]$. ADT induces hot flashes and sleep disturbance [21], and poor sleep disrupts the brain's natural abeta toxin removal process of the glymphatic system [22]. ADT adversely impacts physical activity and function, increases fat mass, reduces lean muscle mass and increases insulin resistance, all of which are indices of metabolic syndrome [17-20]. Insulin resistance, is thought to be a critical AD pathological mechanism in which insulin dysregulation and downstream insulin degrading enzyme impact neuronal death and plaque formation [44, 45]. Transgenic mouse models of $\mathrm{AD}$, fed a high-fat diet inducing both changes in lipids and insulin sensitivity, show a more rapid accumulation of amyloid plaques in brain. This has led to a characterization of $\mathrm{AD}$ as a "type 3" diabetes [46]. ADT may not have a direct causative relationship with $\mathrm{AD}$, however, the initiation of $\mathrm{ADT}$ and related side effects may create a favorable environment for a slow and ongoing disease process of $\mathrm{AD}$ to progress at a more rapid pace [47].

Altered brain metabolism from ADT corresponded to changes in cognition. A positive correlation between performance on a spatial reasoning task (mental rotation) and the posterior cingulate, left inferior parietal lobule (BA40), and left mid temporal lobe (BA39) was observed. A significant decline from baseline performance on this spatial reasoning task was observed in the larger sample on this measure [7]. An inability to orient or to wayfind is a common early symptom in $\mathrm{AD}$ patients, with frequent incidents of patients getting lost. In addition, AD patients perform poorly on tasks of spatial orientation, and learning that is not entirely accounted for by impairments in memory and new learning $[48,49]$.

A positive association was found between a measure of executive function (Stroop test) and middle frontal gyrus (BA 6) and precuneus (Fig. 2). Other studies have shown an association between frontal region activation and the Stroop test [50] as well as a decline in executive functions from ADT [8]. Declines in executive function are often prominent along with memory declines in AD patients as well as in type-2 diabetes [43, 51].

In humans, there is some indication of a decline of neural activation from ADT in frontal regions while performing a working memory task as measured by functional magnetic resonance imaging. Chao et al. [52] reported decreased medial prefrontal cortical activation during a working memory task, as well as decreased connectivity between the medial prefrontal cortex and other regions in men on ADT compared to controls. 
Our findings revealed a negative association for two verbal memory tasks (story recall and verbal list learning) (Fig. 3), indicating that a decrease in glucose metabolism during ADT was associated with better performance on these tasks. Prior studies have reported mixed findings with regard to verbal memory changes in men undergoing ADT including both improved verbal memory [9] as well as significant declines [53].

Pre-post changes in the thalamus and negative associations between the hypothalamus and brainstem and mood were also observed, suggesting that lower metabolic function was associated with endorsement of higher levels of depression, irritability, and anxiety. While the role of the hypothalamus in integrating limbic functions is well characterized [54], the association with more subtle changes in self-endorsed mood has not been previously reported. Testosterone has been shown to have an anxiolytic effect in animals, and our results suggest a connection between the hypothalamus and mood symptoms. Animal models generally support a connection between the hypothalamus and aggression. Men undergoing ADT have been shown to endorse significant mood symptoms including depression, anxiety, and irritability [55].

Findings from this preliminary study must be interpreted with caution due to the limited sample. Results should be replicated with more subjects. Nonetheless, despite these limitations, our study has several strengths including a within subjects, repeated measure design with on treatment compared to off treatment baseline. Thus, although the absolute sample size is small, the within participant comparison adds strength to the analysis. Further, the study establishment of a pre-ADT baseline, which is challenging in clinical studies given a patient's desire to rapidly initiate treatment once a rise in PSA (biochemical relapse) has been discovered. Men were carefully screened to ensure no evidence of metastatic disease, and were administered a consistent, predefined ADT regimen (leuprolide acetate and flutamide) and thorough evaluations of both cognition and mood concurrently with neuroimaging. This is in contrast to some studies of ADT that have included men with various anti-androgen treatment regimens including orchiectomy or have included men with varying time exposure to ADT (months to years), or evaluated men after the initiation of ADT.

\section{Conclusion}

Our results indicate changes in brain activity as measured by FDG-PET imaging in men with prostate cancer undergoing androgen deprivation, compared to their preandrogen deprivation baseline. These changes occur in brain regions associated with mood and higher-order cognitive processing and were associated with behavioral measures of mood and cognition. Associations between hypothalamus and mood measures may help explain prior findings of increased irritability and mood changes in men with idiopathic hypogonadism (low testosterone) as well as mood changes with androgen deprivation. The overlap of brain regions with metabolic decline in ADT and early AD and diabetes suggests possible common mechanisms.

\section{Disclaimer}

Views and opinions of, and endorsements by the author(s) do not reflect those of the US Army or the Department of Defense.

Acknowledgements We thank Marisa Johnson for excellent technical support. This study was supported in part by NCI CA120933, NIA K01AG00858, M01-RR-00037, and Department of Defense, Prostate Cancer Research Program (DAMD17-03-1-0045).

\section{Compliance with ethical standards}

Conflict of interest C.S.H. has acted in a consulting/advisory role for Dendreon, Bayer, Medivation, Ferring, Orion, Emergent Biosolutions, MorphoSys, Churchill Pharmaceuticals, Astellas Pharma, Clovis Oncology, Parexel, Asana Biosciences, Endocyte, Blue Earth Diagnostics; has received research funding for projects (unrelated to this project) from Algeta/Bayer, Aragon Pharmaceuticals, AstraZeneca, Dendreon, Genentech, Medivation, Sanofi, Emergent Biosolutions, Bayer, Pfizer; has received travel accomodations or expenses from Bayer, Dendreon, Astellas Pharma, Emergent Biosolutions, Churchill Pharmaceuticals, Clovis Oncology, Blue Earth Diagnostics, Asana Biosciences, Emergent Biosolutions, Endocyte, Ferring, Genentech, Orion Pharma GmbH, Menarini, Myriad Genetics. The remaining authors declare that they have no conflict of interest.

Open Access This article is licensed under a Creative Commons Attribution-NonCommercial-NoDerivatives 4.0 International License, which permits any non-commercial use, sharing, distribution and reproduction in any medium or format, as long as you give appropriate credit to the original author(s) and the source, and provide a link to the Creative Commons license. You do not have permission under this license to share adapted material derived from this article or parts of it. The images or other third party material in this article are included in the article's Creative Commons license, unless indicated otherwise in a credit line to the material. If material is not included in the article's Creative Commons license and your intended use is not permitted by statutory regulation or exceeds the permitted use, you will need to obtain permission directly from the copyright holder. To view a copy of this license, visit http://creativecommons.org/licenses/by-nc-nd/4.0/.

\section{References}

1. Higano C. Androgen deprivation therapy: monitoring and managing the complications. Hematol Oncol Clin North Am. 2006;20:909-23.

2. Joly F, Alibhai SM, Galica J, Park A, Yi QL, Wagner L, et al. Impact of androgen deprivation therapy on physical and cognitive function, as well as quality of life of patients with nonmetastatic prostate cancer. J Urol. 2006;176(6 Pt 1):2443-7. 
3. Nead KT, Gaskin G, Chester C, Swisher-McClure S, Dudley JT, Leeper NJ, et al. Androgen deprivation therapy and future Alzheimer's disease risk. J Clin Oncol. 2016;34:566-71.

4. Nead KT, Gaskin G, Chester C, Swisher-McClure S, Leeper NJ, Shah NH. Association between androgen deprivation therapy and risk of dementia. JAMA Oncol. 2017;3:49-55.

5. Jhan JH, Yang YH, Chang YH, Guu SJ, Tsai CC. Hormone therapy for prostate cancer increases the risk of Alzheimer's disease: a nationwide 4-year longitudinal cohort study. Aging Male. 2017;20:1-6.

6. Chung SD, Lin HC, Tsai MC, Kao LT, Huang CY, Chen KC. Androgen deprivation therapy did not increase the risk of Alzheimer's and Parkinson's disease in patients with prostate cancer. Andrology. 2016;4:481-5.

7. Cherrier MM, Aubin S, Higano CS. Cognitive and mood changes in men undergoing intermittent combined androgen blockade for non-metastatic prostate cancer. Psychooncology. 2009;18:237-47.

8. Mohile SG, Lacy M, Rodin M, Bylow K, Dale W, Meager MR et al. Cognitive effects of androgen deprivation therapy in an older cohort of men with prostate cancer. Crit Rev Oncol Hematol 2010; 75(2):152-59.

9. Salminen EK, Portin RI, Koskinen A, Helenius H, Nurmi M. Associations between serum testosterone fall and cognitive function in prostate cancer patients. Clin Cancer Res. 2004;10:7575-82.

10. Cherrier MM, Borghesani PR, Shelton AL, Higano CS. Changes in neuronal activation patterns in response to androgen deprivation therapy: a pilot study. BMC Cancer. 2010;10:1.

11. Chao HH, Hu S, Ide JS, Uchio E, Zhang S, Rose M, et al. Effects of androgen deprivation on cerebral morphometry in prostate cancer patients--an exploratory study. PLoS ONE. 2013;8:e72032.

12. Rosario ER, Carroll JC, Pike CJ. Evaluation of the effects of testosterone and luteinizing hormone on regulation of betaamyloid in male 3xTg-AD mice. Brain Res. 2012;1466:137-45.

13. Ding F, Yao J, Zhao L, Mao Z, Chen S, Brinton RD. Ovariectomy induces a shift in fuel availability and metabolism in the hippocampus of the female transgenic model of familial Alzheimer's. PLoS ONE. 2013;8:e59825.

14. Almeida OP, Waterreus A, Spry N, Flicker L, Martins RN. One year follow-up study of the association between chemical castration, sex hormones, beta-amyloid, memory and depression in men. Psychoneuroendocrinology. 2004;29:1071-81.

15. Gandy S, Almeida OP, Fonte J, Lim D, Waterrus A, Spry N, et al. Chemical andropause and amyloid-beta peptide. JAMA. 2001;285:2195-6.

16. Casadesus G, Webber KM, Atwood CS, Pappolla MA, Perry G, Bowen RL, et al. Luteinizing hormone modulates cognition and amyloid-beta deposition in Alzheimer APP transgenic mice. Biochim Biophys Acta. 2006;1762:447-52.

17. Choi SM, Kam SC. Metabolic effects of androgen deprivation therapy. Korean J Urol. 2015;56:12-8.

18. Basaria S. Androgen deprivation therapy, insulin resistance, and cardiovascular mortality: an inconvenient truth. J Androl. 2008;29:534-9.

19. Clay CA, Perera S, Wagner JM, Miller ME, Nelson JB, Greenspan SL. Physical function in men with prostate cancer on androgen deprivation therapy. Phys Ther. 2007;87:1325-33.

20. Galvão DA, Spry NA, Taaffe DR, Newton RU, Stanley J, Shannon $\mathrm{T}$, et al. Changes in muscle, fat and bone mass after 36 weeks of maximal androgen blockade for prostate cancer. BJU Int. 2008;102:44-7.

21. Savard J, Ivers H, Savard MH, Morin CM. Cancer treatments and their side effects are associated with aggravation of insomnia: results of a longitudinal study. Cancer. 2015;121:1703-11.

22. Pistollato F, Sumalla Cano S, Elio I, Masias Vergara M, Giampieri F, Battino M. Associations between sleep, cortisol regulation, and diet: possible implications for the risk of Alzheimer disease. Adv Nutr. 2016;7:679-89.

23. Bohnen NI, Djang DS, Herholz K, Anzai Y, Minoshima S. Effectiveness and safety of 18F-FDG PET in the evaluation of dementia: a review of the recent literature. J Nucl Med. 2012;53:59-71.

24. Cherrier MM, Rose AL, Higano C. The effects of combined androgen blockade on cognitive function during the first cycle of intermittent androgen suppression in patients with prostate cancer. J Urol. 2003;170:1808-11.

25. Kawamichi H, Kikuchi Y, Noriuchi M, Senoo A, Ueno S. Distinct neural correlates underlying two- and three-dimensional mental rotations using three-dimensional objects. Brain Res. 2007; 1144:117-26.

26. Wechsler D. Weschler Adult Intelligence Scale-Revised. San Antonio: The Psychological Corporation; 1981.

27. Vandenberg SG, Kuse AR. Mental rotations, a group test of threedimensional spatial visualization. Percept Mot Skills. 1978;47:599-601.

28. Moscovitch M. Cognitive resources and dual-task interference effects on retrieval in normal people: the role of the frontal lobes and medial temporal cortex. Neuropsychology. 1994;8:524-34.

29. Wechsler D. Wechsler Memory Scale-Revised. San Antonio: The Psychological Corporation; 1987.

30. Spreen O, Strauss E. A compendium of neuropsychological tests. New York: Oxford University Press; 1991.

31. Lezak MD. Neuropsychologcial assessment. 3rd ed. New York: Oxford University Press; 1995.

32. Petrides M, Milner B. Deficits on subject-ordered tasks after frontal and temporal lobe lesions in man. Neuropsychologia. 1982;20:249-62.

33. McNair DM, Lorr M, Droppleman LF. Profile of mood states. San Diego: Educational and Industrial Testing Service; 1992.

34. Talairach J, Tournoux P. Co-planar stereotaxic atlas of the human brain. New York: Thieme Medical Publishers; 1988.

35. Minoshima S, Koeppe RA, Frey KA, Kuhl DE. Anatomic standardization: linear scaling and nonlinear warping of functional brain images. J Nucl Med. 1994;35:1528-37.

36. Minoshima S, Giordani B, Berent S, Frey KA, Foster NL, Kuhl DE. Metabolic reduction in the posterior cingulate cortex in very early Alzheimer's disease. Ann Neurol. 1997;42:85-94.

37. Minoshima S, Frey KA, Koeppe RA, Foster NL, Kuhl DE. A diagnostic approach in Alzheimer's disease using threedimensional stereotactic surface projections of fluorine-18-FDG PET. J Nucl Med. 1995;36:1238-48.

38. Drzezga A, Riemenschneider M, Strassner B, Grimmer T, Peller M, Knoll A, et al. Cerebral glucose metabolism in patients with AD and different APOE genotypes. Neurology. 2005;64:102-7.

39. Worsley KJ, Evans AC, Marrett S, Neelin P. A three-dimensional statistical analysis for CBF activation studies in human brain. $\mathrm{J}$ Cereb Blood Flow Metab. 1992;12:900-18.

40. Peskind ER, Petrie EC, Cross DJ, Pagulayan K, McCraw K, Hoff $\mathrm{D}$, et al. Cerebrocerebellar hypometabolism associated with repetitive blast exposure mild traumatic brain injury in 12 Iraq war Veterans with persistent post-concussive symptoms. Neuroimage. 2011;54(Suppl 1):S76-82.

41. Worsley KJ, Marrett S, Neelin P, Vandal AC, Friston KJ, Evans AC. A unified statistical approach for determining significant signals in images of cerebral activation. Hum Brain Mapp. 1996;4:58-73.

42. Drzezga A, Lautenschlager N, Siebner H, Riemenschneider M, Willoch F, Minoshima S, et al. Cerebral metabolic changes accompanying conversion of mild cognitive impairment into Alzheimer's disease: a PET follow-up study. Eur J Nucl Med Mol Imaging. 2003;30:1104-13. 
43. Chen Y, Liu Z, Zhang J, Xu K, Zhang S, Wei D, et al. Altered brain activation patterns under different working memory loads in patients with type 2 diabetes. Diabetes Care. 2014;37:3157-63.

44. Jolivalt CG, Calcutt NA, Masliah E. Similar pattern of peripheral neuropathy in mouse models of type 1 diabetes and Alzheimer's disease. Neuroscience. 2012;202:405-12.

45. Yeh CW, Yeh SH, Shie FS, Lai WS, Liu HK, Tzeng TT, et al. Impaired cognition and cerebral glucose regulation are associated with astrocyte activation in the parenchyma of metabolically stressed APPswe/PS1dE9 mice. Neurobiol Aging. 2015;36:2984-94.

46. Kandimalla R, Thirumala V, Reddy PH. Is Alzheimer's disease a type 3 diabetes? A critical appraisal. Biochim Biophys Acta. 2017;1863:1078-89.

47. Burnham SC, Bourgeat P, Dore V, Savage G, Brown B, Laws S, et al. Clinical and cognitive trajectories in cognitively healthy elderly individuals with suspected non-Alzheimer's disease pathophysiology (SNAP) or Alzheimer's disease pathology: a longitudinal study. Lancet Neurol. 2016;15:1044-53.

48. Cherrier MM, Mendez M, Perryman K. Route learning performance in Alzheimer disease patients. Neuropsychiatry Neuropsychol Behav Neurol. 2001;14:159-68.

49. Kalová E, Vlcek K, Jarolímová E, Bures J. Allothetic orientation and sequential ordering of places is impaired in early stages of
Alzheimer's disease: corresponding results in real space tests and computer tests. Behav Brain Res. 2005;159:175-86.

50. Volkow ND, Gur RC, Wang GJ, Fowler JS, Moberg PJ, Ding YS, et al. Association between decline in brain dopamine activity with age and cognitive and motor impairment in healthy individuals. Am J Psychiatry. 1998;155:344-9.

51. Kaiser NC, Melrose RJ, Liu C, Sultzer DL, Jimenez E, Su M, et al. Neuropsychological and neuroimaging markers in early versus late-onset Alzheimer's disease. Am J Alzheimers Dis Other Demen. 2012;27:520-9.

52. Chao HH, Uchio E, Zhang S, Hu S, Bednarski SR, Luo X, et al. Effects of androgen deprivation on brain function in prostate cancer patients - a prospective observational cohort analysis. BMC Cancer. 2012;12:371.

53. Green HJ, Pakenham KI, Headley BC, Yaxley J, Nicol DL, Mactaggart PN, et al. Altered cognitive function in men treated for prostate cancer with luteinizing hormone-releasing hormone analogues and cyproterone acetate: a randomized controlled trial. BJU Int. 2002;90:427-32.

54. Schindler S, Geyer S, Strauss M, Anwander A, Hegerl U, Turner $\mathrm{R}$, et al. Structural studies of the hypothalamus and its nuclei in mood disorders. Psychiatry Res. 2012;201:1-9.

55. Pirl WF, Siegel GI, Goode MJ, Smith MR. Depression in men receiving androgen deprivation therapy for prostate cancer: a pilot study. Psychooncology. 2002;11:518-23. 